petriger Säure kaum etwas gelblich; aus den zwischenliegenden Farbennüancen lässt sich auf eine grössere oder geringere Beimischung des Benzols zum Benzin schliessen.

Für die Neubearbeitung der Pharmacopöe ergiebt sich aus Vorstehendem, dass die Prüfung des Benzin mit ammoniakalischer Silberlösung wenig Werth hat, dass dagegen die Löslichkeit des Benzin im Weingeist genau mit Zahlen zu fixiren ist; hinzugefügt kömnte noch werden, dass Jod vom Benzin mit anilinrother Farbe gelöst wird und dass Benzin mit rauchender Salpetersäure vermischt nur eine ganz schwache Farbenveränderung ins Gelbliche erleiden darf.

Zum Schlusse möchte ich mir noch erlauben darauf hin«uwcisen, wie schlecht sich in einer Pharmacopöe neuen Datums der Fortgebrauch des Namens "Aether Petrolei" ausnehrnen wird. Man müge für diesen Artikel lieber ganz willkürlich einen Namen wählen (Rhigolen oder dergl.), als von Aether sprechen, wo in chemischer Beziehung nicht die geringste Achnlichkeit mit einem solchen vorhanden ist.

\title{
Pharmacognostische Notizen.
}

Vou Carl IIartwieh, Apotheker.

\section{Chinesische Gallen.}

Unter dem Namen „Birnengallen " findet sich seit einiger Zeit eine Sorte chinesischer Gallen im Handel, deren von den gewöhnlichen etwas abweichonde Gestalt zu einer Untersuchung reizte. Die unterscheidenden Merkmale, die ich fand, sind jedes fuir sich nicht bedeutend, doch waren sie bei allen von mir untersuchten Stücken so bestündig, dass ich wohl glaube, die fraglichen Gallen ihrer Abstammung nach als ron den. gewöhnlichen versclieden bezeichnen zu dürfon.

Dic „Birnengallen" sind höchstens von der Grösse einer mässigen Pflaume und zeigen nie dic eigenthümliche, so charakteristische Zackenbildung, sind aber fast immer an der Spitze umgrebogen. Dic Behaarung ist eine sehr spürliche, 
so dass man oft ganze Reihen mikroskopischer Präparate erhält, an denen nicht cin einziges Haar sich findet. 1)ie Haare sind unverzweigt und bestehen oft ans 2-4 Zellen (sie gleichen denen der gewöhnlichen Rhus-Gallen völlig, also auch diese sind nicht immer einzellig, wie sie Flückiger in den "Grundlagen der pharmaceutischen Waarenkunde abbildet). Im Querschnitt folgt auf die Epidermis eine Schicht stark tangential gestreckten Parenchymgewebes, die sich aus mindestens 7-8 Zullreihen zusammensetzt. Diese tangentiale Streckung der Zellen nimmt von hier bis zur Mitte stetig $a b$, wo sie ziemlich plötzlich $\%$ einer ausgesprochen radialen wird. (Bei den gewöhnlichen chinesischen Gallen ist die unter der Epidermis befindliche stark tangential gestreckte Schicht aus höchstens drei Zellreihen zusammengesetzt und die radiale Streckung in der zweiteu Hält'te tritt nie cin.)

Durch die ganze Masse zerstreut liegen die stattlichen Milchsaftschläuche, die gregen die innere Wand an Zahl zu-, aber an Grösse abnehmon. Sie sind von einem mehr oder weniger deullichen Ring kleinerer Parenchynzellen ungeben nnd aus einem erweiterten Intercellularraum hervorgegangen. Fast ausnahmslos unter jedem liegt ein meist nicht starker Fibrovasalstrang, mit jenem durch ein Bastbündel verbunden, sehr selten erstreckt sich der Bast um den Milchsuftschlauch völlig herum. Die Gefässe bestehen ans abrollbaren Spiroïden. Unmittelbar an der Innenwand verschwinden die Milchsaftschläuche und es sind hier die Gefässbündel zu cinem fest znsammunhängenden Ring vereinigt. Die letzte Zellschicht der Innenseite besteht wie die Epidermis aus fast kubischen Zellen. Nach Entfernung der Gerbsäure findet man in den Parenchymzellen kleine Amylumkörner in grosser Menge. Die zuletzt geschilderten anatomischen Verhältnisse, nämlich die Beschaffenheit der Milchsaftgefässe und ihre enge Zusammengehörigkeit mit den Fibrovasalsträngen, stimmen bei beiden Gallensorten völlig überein, höchstens ist bei den grewöhnlichen ehinesischen Gallen der den Milchsaftschlauch umgebende Ring kleiner Zellen oft dentlicher ausgebildet; ich habe aber doch geglaubt, darauf eingehen zu müssen, da in 

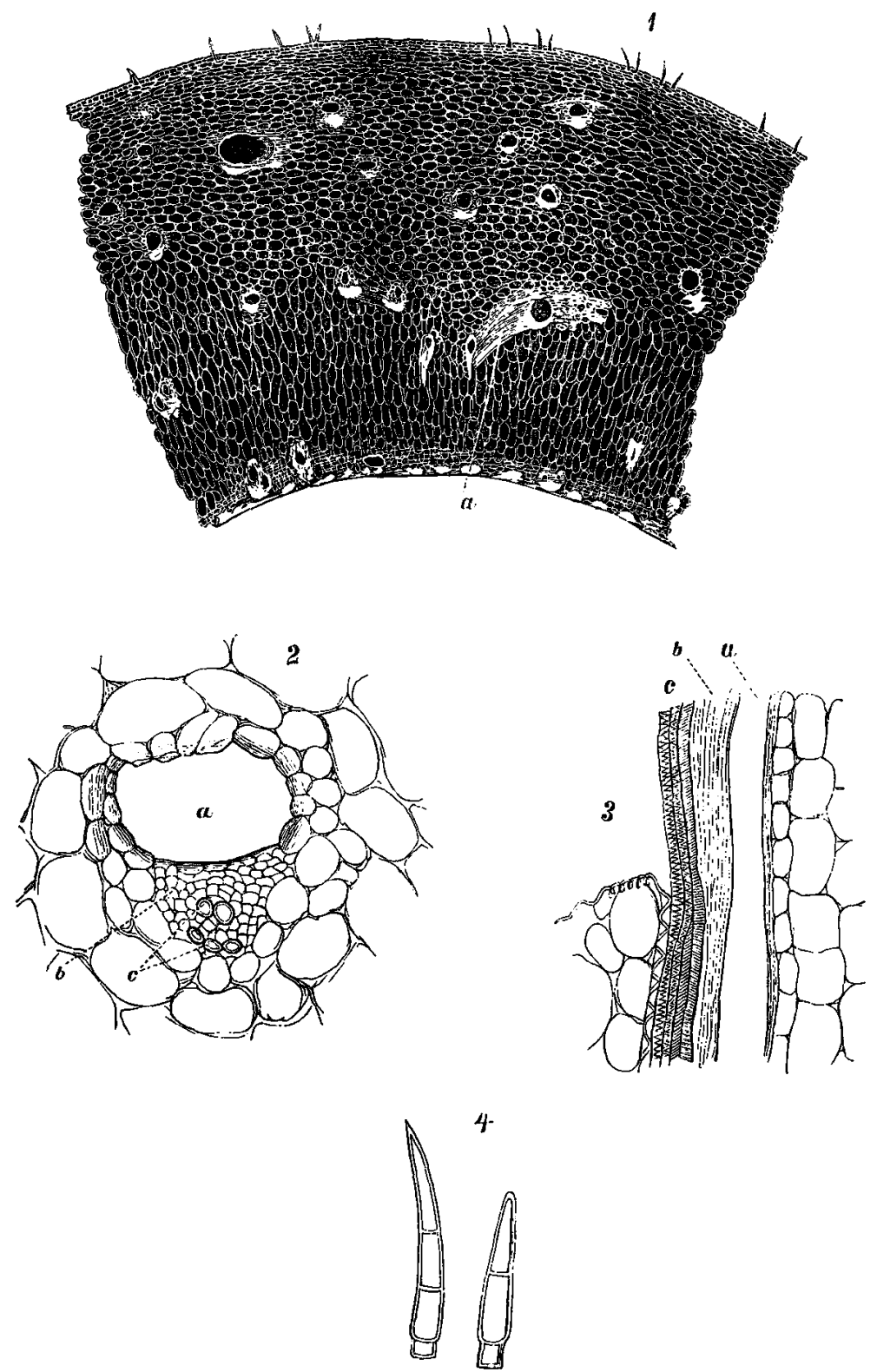
manchen Handbüchern der Pharmacognosie diese Verhältnisse nicht genauer geschildert werden.

Der Gehalt an Gerbsäure ist ein sehr bedeutender, ich fand nach der Löwentlıal'schen Nethode durchschnittlich $72,0 \%$, er blcibt also hinter dem der besten chinesischen Gallen kaum zurück.

Leider habe ich ubor die botanische Abstammung der "Birnengallen" Nichts in Erfahrung bringen können, aber ich glaube, dass anch ein so unvollständiger Beitrarr, wie ich ihn zu liefern vermochte, bei dieser interessanten Droge nicht ganz ohne Wichtigkeit ist.

\section{Erklärung der Figuren.}

1. Querschnitt durch cine Birnengalle, bei $a$ ist ein horizontal verlaufender Milchsaftschlauch getrofien.

2. Ein Milchsaftschlauch mit Fibrovasalstrang, stärker vergrössert; $a$. der Milchsaftschlauch; $b$. der Bast; $c$. Gefässe.

3. längsschnitt durch einen Milchsaftschlanch; $a, b, c$ wie in 2.

4. Zwei Haare von der Epidermis.

\section{Gambir.}

Als Gambir-Catechu findet man meist nur einc Sorte erwähnt, nämlich dic in grösseren oder kleineren Würfeln. Da dieses Extract aber in neuerer Zeit grössere Anwendung in der Technik findet, so wird es auch vielfach in anderer Form ausgeführt, wie dies auch Flückiger in der "pharmacognostischen Wanderung durch die Pariser Ausstellung “ erwähnt.

Ueber eine solche neuc Sorte, die mir in letzter Zeit vielfach zu Gesicht gekommen, will ich kurz berichten: Dieses Gambir kommt in grossen, oblongen, 2 Ctr. schweren Blöcken in den Handel, die, wie auch meist das AcacienCatechu, in Matten verpackt sind. Die Blöcke sind aussen ziemlich reichlich mit Sägespähnen bestreut. Auf dem Bruche erscheint die Masse matt, heller und dunkler braun marmorirt, wenig lückig. Als echtes Gambir charakterisirt es sich unter dem Mikroskop durch den ungemein grossen Inhalt 
an Catechinkrystallen, neben diesen findet man spärliche Pflanzenreste und quadratische Krystalle von Calciumoxalat. Der Gehali an Gerbstoff beträgt $45-46 \%$. Es kommt aus Calcutta in den Handel.

\section{Ueber das Alkaloïd der Granatwurzelrinde.}

Von Dr. mod. Ferd. A ug. Falck, Prof. in Kiel.

In ihren Sitzungen vom 20. Mai und 26. August 1878 wurden der französischen Academic der Wissenschaften Correspondenzen vorgelegt, welche den Titel führen: Sur la pelletiérine, alcaloide de l'écorce de grenadier. Note de: M. Tanret, présentée par M. Berthelot. (Compt. rend. 861279,87358 ).

Tanret theilt mit, dass es ihm gelungen ist, aus der Rinde der Wurzel und Stengel des Granatbaums, durch Behandlung mit Kalkmilch, Ausziehen mit Wasser, Schütteln mit Chloroform und Behandeln der Chloroformlösung mit verdünnten Säuren, cin Salz in Krystallen darzustellen, aus welchem durch Zersetzen mit kohlensaurem Kalium ein Alkalo id isolirt werden konnte.

Dieses Alkaloid ist ölartig, farblos, bei gewöhnlicher Temperatur schon flüchtig; es siedet bei $180^{\circ} \mathrm{C}$., löst sich leicht in Wasser, Alkohol, Aether, Chloroform; es hat bei $0^{\circ} \mathrm{C}$. ein specifisches Gewicht $=0,999$, ein Rotationsvermögen : $(\alpha) \mathrm{i}=+8^{\circ}$. Seine Zusammensetzung führt zu der Formel: $\mathrm{C}^{8} \mathrm{H}^{13} \mathrm{NO}$ (Dampfdichte gef:: 4,66; ber.: 4,81). Das Alkaloid reagirt stark alkalisch, bildet bei Gegenwart von Säuredïmpten, ähnlich dem Ammoniak, dicke Nebel, fällt Metallsalze, Tannin, Jodkaliumdoppelsalze, Phosphormolybdänsäure; tärbt sich mit Schwetelsäure und Kaliumbichromat: grün; liefert krystallisirende Salze.

Die Ausbeute betrug aus $1 \mathrm{Kg}$. Rinde des Handels: $4 \mathrm{~g}$. schwetelsaures Salz.

Tanret nennt dieses Alkaloïd, wolchem nach einigen therapeutischen Untersuchungen die Wirkung der Granal- 